\title{
Pathogenic analysis of sputum from ventilator-associated pneumonia in a pediatric intensive care unit
}

\author{
BO-TAO NING, CHEN-MEI ZHANG, TAO LIU, SHENG YE, ZI-HAO YANG and ZHEN-JIE CHEN \\ Department of Pediatric Intensive Care Unit, Children's Hospital, School of Medicine, \\ Zhejiang University, Hangzhou, Zhejiang 310003, P.R. China
}

Received August 26, 2012; Accepted October 12, 2012

DOI: $10.3892 /$ etm.2012.757

\begin{abstract}
Ventilator-associated pneumonia (VAP) is a common and sometimes fatal complication in pediatric intensive care units (PICU). The aim of our study was to characterize the distribution and drug susceptibility of the pathogens isolated from the sputum of patients with VAP in the PICU of our hospital and to provide support to the administration of antibiotics early and reasonably in the clinic. Our study was conducted between January 2007 and December 2011 at the PICU of the Children's Hospital of Zhejiang University School of Medicine. The endotracheal aspirates were collected and transported to a microbiology laboratory within $15 \mathrm{~min}$. The pathogens were routinely analyzed and identified with Vitek 60 and Kirby-Bauer disk diffusion methods. Among the 121 VAP patients, 127 pathogenic strains were isolated from sputum specimens. Gram-negative and gram-positive bacteria and fungi accounted for $64.57 \%$ (82/127), 29.92\% (38/127) and $5.51 \%$ (7/127), respectively. Acinetobacter baumannii (25.61\%), Escherichia coli (20.27\%), Stenotrophomonas maltophilia (20.27\%), Klebsiella pneumoniae (16.22\%) and Pseudomonas aeruginosa (9.46\%) were frequently identified isolates among gram-negative bacteria. Staphylococci were susceptible to vancomycin and linezolid. All fungi were sensitive to the antimicrobial agents. The gram-negative bacteria were more prevalent than gram-positive bacteria and fungi in VAP and demonstrated a higher drug resistance. It is important to administer antimicrobial agents early and reasonably for children with VAP. Knowledge of antibiotic resistance and the characteristics of drug resistance is important for VAP prophylaxis and treatment.
\end{abstract}

Correspondence to: Professor Chen-Mei Zhang, Department of Pediatric Intensive Care Unit, Children's Hospital, School of Medicine, Zhejiang University, 57 Zhugan Lane, Hangzhou, Zhejiang 310003, P.R. China

E-mail: chzcm@zju.edu.cn

Key words: pediatric intensive care unit, ventilator associated pneumonia, sputum, drug susceptibility

\section{Introduction}

Children in the pediatric intensive care unit (PICU) are at high risk of hospital-acquired infection. Ventilator-associated pneumonia (VAP) is the most common hospital-acquired infection, with an incidence of $8-28 \%(1,2)$. VAP is becoming a common and fatal complication as mechanical ventilation is being increasingly used in the PICU. VAP is defined as pneumonia occurring $>48 \mathrm{~h}$ after patients have accepted intubation and received mechanical ventilation. It often presents as deteriorated pneumonia or as new foci in the lungs. The occurrence rate in adults reaches $9-27 \%$ and the mortality rate is $20-50 \%$ (3-5). In children, due to their poor immunity, serious original disease and prolonged mechanical ventilation, the mortality rate of $24-76 \%$ is higher than in adults $(1,2)$. In order to study the epidemiology and changes in antibacterial susceptibility to allow guidance of the empirical and reasonable use of antibiotics to reduce the mortality of children with VAP, we retrospectively studied the pathogenic bacteria distribution and drug resistance in 121 VAP patients in the PICU of the Children's Hospital affiliated to Zhejiang University from January 2007 to December 2011.

\section{Patients and methods}

Patients. From January 2007 to December 2011, a total of 492 patients underwent mechanical ventilation and of these, 121 patients developed VAP. From these patients 127 strains were isolated. The general data of the 121 children (whose ages ranged from 2 months to 16 years, 3 months; median age 3 years, 6 months) are shown in Table I. The present study was approved by the ethics committee of the Children's Hospital of Zhejiang University School of Medicine and informed consent was obtained from all patients.

Definition of VAP. The diagnostic criteria of VAP are as follows: i) pneumonia occurring more than $48 \mathrm{~h}$ after intubation and mechanical ventilation; ii) two or more of the following four criteria: a) fever $>38.3^{\circ} \mathrm{C}$; b) leukocytosis $>10,000$ cells $/ \mathrm{ml}$ or $<5,000$ cells $/ \mathrm{ml}$; c) purulent tracheobronchial secretion; and d) new pathogenic bacteria isolated from bronchial secretions; iii) a new and persistent ( $>48 \mathrm{~h}$ ) infiltrate on chest radiograph (6).

Antibiotic susceptibility test. Bacteria were identified using a Vitek-60 automated analysis system which was purchased from 
Table I. General data of the121 VAP patients.

\begin{tabular}{lcc}
\hline Variables & Number of patients & Rate $(\%)$ \\
\hline Gender & 75 & \\
Male & 46 & 61.98 \\
Female & & 38.02 \\
VAP occurrence & 36 & \\
$<5$ days & 85 & 29.75 \\
$\geq 5$ days & & 70.25 \\
Basic diseases & 39 & \\
Severe pneumonia & 17 & 32.23 \\
Sepsis with ARDS & & 14.05 \\
Central nervous & 29 & \\
system infection & 11 & 23.97 \\
Trauma & 9 & 9.09 \\
Drowning & 7 & 7.44 \\
Drug poisoning & 3 & 5.79 \\
Epilepsy & 2 & 2.48 \\
Asthma & 4 & 1.65 \\
Non-traumatic & 4.31 \\
intracranial hemorrhage & & \\
\hline VAP ver & & \\
\hline
\end{tabular}

VAP, ventilator-associated pneumonia; ARDS, acute respiratory distress syndrome.

bioMérieux (Durham, UK). Susceptibility paper and susceptibility medium (MH medium) were purchased from Oxiod Ltd. (Basingstoke, UK) and Marcel Mérieux, respectively. Under sterile conditions, a disposable sterile sputum collector was used to collect secretion samples from the trachea through an endotracheal intubation catheter. A total of 127 sputum samples were cultured and a routine drug susceptibility test was performed within 15 min using the Vitek-60 analysis system and Kirby-Bauer disk diffusion method. The extended-spectrum $\beta$-lactamase (ESBL) strains were detected by double disk test. If more than two consecutive results were the same in one patient, we recorded the results of the sputum culture once; if the results were different, we recorded each result of the sputum culture.

Statistical analysis. All data were analyzed using Stata software, version 9.0. Measurement data are denoted by median values and numeration data were presented as rate and constituent ratio.

\section{Results}

Distribution of pathogens. The incidence of VAP was $24.59 \%$ (121/492). A total of 127 strains were detected from 121 VAP patients. Six patients were diagnosed with mixed infections. None were recurrent. Gram-negative bacteria, gram-positive bacteria and fungi accounted for $64.57 \%(82 / 127)$, $29.92 \%(38 / 127)$ and $5.51 \%$ (7/127) of the strains, respectively. The most common pathogens were Acinetobacter baumannii, Escherichia coli, Stenotrophomonas maltophilia, Klebsiella pneumoniae and Pseudomonas aeruginosa (Table II).
Table II. Distribution of detected pathogens in the sputum of children with VAP.

\begin{tabular}{lcc}
\hline Pathogen & $\begin{array}{c}\text { Number } \\
\text { of strains }\end{array}$ & $\begin{array}{c}\text { Proportion } \\
(\%)\end{array}$ \\
\hline Gram-negative bacteria & 82 & 64.57 \\
Acinetobacter baumannii & 21 & 25.61 \\
Escherichia coli & 17 & 20.27 \\
Stenotrophomonas maltophilia & 17 & 20.27 \\
Klebsiella pneumoniae & 12 & 16.22 \\
Pseudomonas aeruginosa & 11 & 9.46 \\
Sphingomonas paucimobilis & 2 & 2.70 \\
Aeromonas hydrophila & 1 & 1.35 \\
Salmonella thompson & 1 & 1.35 \\
Gram-positive bacteria & 38 & 29.92 \\
Staphylococcus epidermidis & 10 & 26.32 \\
Other CoNS & 9 & 23.68 \\
Streptococcus & 7 & 18.42 \\
Staphylococcus aureus & 6 & 15.79 \\
Enterococcus & 4 & 10.53 \\
Micrococcus & 1 & 2.63 \\
Bacillus & 1 & 2.63 \\
Fungus & 7 & 5.51 \\
Candida albicans & 7 & 5.51 \\
Total & 127 & 100.00 \\
& &
\end{tabular}

VAP, ventilator-associated pneumonia; CoNS, coagulase negative staphylococcus.

Drug resistance results of common pathogens. The detection rates of ESBL-producing Escherichia coli, ESBL-expressing Klebsiella pneumoniae and methicillin-resistant Staphylococcus aureus (MRSA) were 58.82\% (10/17), 41.67\% (5/12) and 66.67\% (4/6), respectively.

The gram-negative bacilli demonstrated multiple drug resistance. The 5 most commonly isolated strains were Acinetobacter baumannii, Escherichia coli, Stenotrophomonas maltophilia, Klebsiella pneumoniae and Pseudomonas aeruginosa. All of them, with the exception of Acinetobacter baumannii, were sensitive to cefoperazone/sulbactam. Escherichia coli and Klebsiella pneumoniae demonstrated no drug resistance to meropenem and imipenem; however, they had a high resistance to most penicillins and cephalosporins. All Pseudomonas aeruginosa isolates were sensitive to cefoperazone/sulbactam, imipenem, piperacillin/tazobactam, amikacin and levofloxacin; however, the drug resistance to meropenem was high $(72.73 \%)$. All Stenotrophomonas maltophilia isolates were sensitive to cefoperazone/sulbactam, piperacillin/tazobactam and levofloxacin; $35.29 \%$ of them were resistant to cefoxitin and its drug resistance rate to other antibiotics was $>70 \%$ (Table III).

Gram-positive cocci had no drug resistance to vancomycin and linezolid; however, they demonstrated high drug resistance to penicillin-G, erythromycin, clindamycin and sulfamethoxazole. The positive percentage of $\beta$-lactamase was $76.32 \%$ 
Table III. Antimicrobial resistance patterns of common gram-negative bacteria isolated from the sputum of children with VAP.

\begin{tabular}{|c|c|c|c|c|c|}
\hline \multirow[b]{2}{*}{ Drugs } & \multicolumn{5}{|c|}{ Pathogens (drug resistance rate, \%) } \\
\hline & $\begin{array}{c}\text { Acinetobacter } \\
\text { baumannii } \\
\text { (21 strains) }\end{array}$ & $\begin{array}{c}\text { Escherichia } \\
\text { coli } \\
\text { (17 strains) }\end{array}$ & $\begin{array}{c}\text { Stenotrophomonas } \\
\text { maltophilia } \\
\text { (17 strains) }\end{array}$ & $\begin{array}{c}\text { Klebsiella } \\
\text { pneumoniae } \\
\text { (12 strains) }\end{array}$ & $\begin{array}{c}\text { Pseudomonas } \\
\text { aeruginosa } \\
\text { (11 strains) }\end{array}$ \\
\hline Cefoperazone/sulbactam & 57.14 & 0.00 & 0.00 & 0.00 & 0.00 \\
\hline Cephazoline & 100.00 & 100.00 & 100.00 & 100.00 & 100.00 \\
\hline Cefoxitin & 100.00 & 50.00 & 35.29 & 50.00 & 100.00 \\
\hline Amikacin & 80.95 & 11.76 & 100.00 & 0.00 & 0.00 \\
\hline Levofloxacin & 42.86 & 35.29 & 0.00 & 25.00 & 0.00 \\
\hline Cefuroxime & 100.00 & 82.35 & 100.00 & 100.00 & 100.00 \\
\hline Ceftazidime & 100.00 & 70.59 & 100.00 & 100.00 & 100.00 \\
\hline Ampicillin & 100.00 & 100.00 & 100.00 & 100.00 & 100.00 \\
\hline Cefpiramide & 100.00 & 76.47 & 100.00 & 100.00 & 0.00 \\
\hline Gentamicin & 100.00 & 82.35 & 100.00 & 50.00 & 0.00 \\
\hline Imipenem & 100.00 & 0.00 & 70.59 & 0.00 & 0.00 \\
\hline Meropenem & 100.00 & 0.00 & 100.00 & 0.00 & 72.73 \\
\hline Cefotaxime & 61.90 & 70.59 & 100.00 & 41.67 & 0.00 \\
\hline Piperacillin/tazobactam & 80.95 & 0.00 & 0.00 & 25.00 & 0.00 \\
\hline
\end{tabular}

VAP, ventilator-associated pneumonia.

Table IV. Antimicrobial resistance patterns of staphylococci isolated from the sputum of children with VAP.

\begin{tabular}{|c|c|c|c|}
\hline \multirow[b]{2}{*}{ Drugs } & \multicolumn{3}{|c|}{ Pathogens (drug resistance rate, \%) } \\
\hline & $\begin{array}{c}\text { Staphylococcus epidermidis } \\
\text { (10 strains) }\end{array}$ & $\begin{array}{l}\text { Other CoNS } \\
\text { (9 strains) }\end{array}$ & $\begin{array}{c}\text { Staphylococcus aureus } \\
\text { (6 strains) }\end{array}$ \\
\hline Clindamycin & 60.00 & 44.44 & 50.00 \\
\hline Linezolid & 0.00 & 0.00 & 0.00 \\
\hline Ampicillin/sulbactam & 100.00 & 77.78 & 0.00 \\
\hline Gentamicin & 40.00 & 44.44 & 33.33 \\
\hline Oxacillin & 100.00 & 88.89 & 0.00 \\
\hline Rifampicin & 20.00 & 11.11 & 0.00 \\
\hline Sulfamethoxazole & 90.00 & 55.56 & 66.67 \\
\hline Vancomycin & 0.00 & 0.00 & 0.00 \\
\hline Moxifloxacin & 0.00 & 0.00 & 0.00 \\
\hline Erythromycin & 90.00 & 55.56 & 83.33 \\
\hline Furantoin & 0.00 & 0.00 & 0.00 \\
\hline Levofloxacin & 0.00 & 33.33 & 33.33 \\
\hline Penicillin-G & 100.00 & 88.89 & 83.33 \\
\hline Tetracycline & 40.00 & 22.22 & 0.00 \\
\hline
\end{tabular}

VAP, ventilator-associated pneumonia; CoNS, coagulase negative staphylococcus.

(29/38) in 38 staphylococci. The 10 Staphylococcus epidermidis strains were resistant to oxacillin. The resistance to oxacillin was $88.89 \%$ (8/9) in other coagulase negative staphylococcus (CoNS) strains; however, all 6 Staphylococcus aureus strains demonstrated no drug resistance to oxacillin (Table IV). The 7 strains of Candida albicans were all susceptible to fluconazole.

\section{Discussion}

The PICU is the centre of intensive care and treatment for critically ill children and invasive and complex techniques and equipment, including tracheal intubation and mechanical ventilation, are used frequently. Hamid et al (2) reported that 
VAP occurrence was closely associated with the following factors: i) age of the patient $<1$ year; ii) emergent intubation and iii) persistent and long-time sedation. Patients in the PICU often suffer from severe pathophysiological disorders and hypo-immunologic function. Intubation and mechanical ventilation are essential to maintain normal ventilation and gas exchange. The ventilator guarantees normal oxygenation; however, the airway mucosal destruction and barotrauma increases the risk of pathogenic colonization and infection in the respiratory system. We separated 127 strains of pathogens from 121 sputum samples collected from the lower respiratory tract. Gram-negative bacteria were the dominant pathogens and among them, the most frequent pathogens were Acinetobacter baumannii, Escherichia coli, Stenotrophomonas maltophilia, Klebsiella pneumoniae and Pseudomonas aeruginosa. Staphylococcus epidermidis was the most common grampositive pathogen.

In recent years, the non-fermenters such as Acinetobacter baumannii and Stenotrophomonas maltophilia, have become increasingly prevalent. They have been the main pathogenic bacteria in hospital-acquired infection, particularly in $\operatorname{VAP}(7,8)$. We report that non-fermenters accounted for $46.34 \%$ of the cases of VAP and among them the percentage of Acinetobacter baumannii was $25.61 \%$. High drug resistance often occurred with Acinetobacter baumanii due to the high expression of AmpC enzyme, synthesis of OXA-23 carbapenemases, downregulation of the sensitivity of the efflux pump and production of penicillin-binding protein (PBP). At the same time, certain drug-resistant bacteria may transfer drug-resistant plasmids between each other, resulting in multi-drug resistance $(9,10)$. The current study demonstrated that Acinetobacter baumannii is only partly sensitive to aminoglycosides, quinolones, cefotaxime, piperacillin/tazobactam and cefoperazone/sulbactam and $100 \%$ resistant to carbapenems. Therefore, imipenem and meropenem are not the preferred antibiotics for Acinetobacter baumannii. The drug resistance rates to imipenem and meropenem were higher in other non-fermenters. The resistance rates of Pseudomonas aeruginosa to imipenem and meropenem were 0 and $72.73 \%$, respectively, and in Stenotrophomonas maltophilia, they were 70.59 and $100 \%$, respectively. These results indicate that there may be a natural drug resistance to these two antibiotics $(11,12)$. Therefore, it is necessary to stringently manage and control the use of carbapenems due to the decreased sensitivity of Acinetobacter baumannii, increased resistance of Pseudomonas aeruginosa and the natural drug resistance of Stenotrophomonas maltophilia.

The wide usage of $\beta$-lactam antibiotics in the clinic induces the production of ESBL in Escherichia coli and Klebsiella pneumoniae. ESBL may hydrolyze cephalosporin and transfect between bacteria, which promotes the transmission of drug resistance between pathogens (13). The present study revealed that the percentages of ESBL in Escherichia coli and Klebsiella pneumoniae were 58.82 and $41.67 \%$, respectively. Although they were sensitive to carbapenems, aminoglycosides, quinolones and antibiotics combined with an enzyme inhibitor, they were highly resistant to penicillin and cephalosporin. The gram-positive coccus gained high resistance to penicillin; however, they were completely sensitive to vancomycin and linezolid. Therefore, vancomycin and linezolid are preferred in severe infection caused by Staphylococcus aureus and $\beta$-lactam, sulfamethoxazole, erythromycin and clindamycin antibiotics are no longer recommended in the treatment of severe gram-positive cocci infection $(14,15)$.

Fungal infection accounted for $5.51 \%$ of the cases of VAP in the current study. Candida albicans was the prime pathogen identified in fungal VAP and was sensitive to the majority of antifungal drugs. The factors that may be associated with fungal infections in children are low immune function, long-term usage of broad-spectrum antibiotics, unreasonable usage of glucocorticoids and invasive surgery $(16,17)$.

VAP acutely threatens patient survival in the PICU. Its pathogenic distribution and drug susceptibility are diverse across different centers; therefore, once VAP is diagnosed, the first choice is empirical antibiotic treatment according to previous data. As studies of drug susceptibility are reported, the choice of antibiotic may be performed more selectively.

\section{Acknowledgements}

The study was supported by National Natural Science Foundation of China (No. 30901327).

\section{References}

1. Chastre J and Fagon JY: Ventilator-associated pneumonia. Am J Respir Crit Care Med 165: 867-903, 2002.

2. Hamid MH, Malik MA, Masood J, Zia A and Ahmad TM: Ventilator-associated pneumonia in children. J Coll Physicians Surg Pak 22: 155-158, 2012.

3. Cunnion KM, Weber DJ, Broadhead WE, Hanson LC, Pieper CF and Rutala WA: Risk factors for nosocomial pneumonia: comparing adult critical-care populations. Am J Respir Crit Care Med 153: 158-162, 1996.

4. Kirschenbaum L, Azzi E, Sfeir T, Tietjen P and Astiz M: Effect of continuous lateral rotational therapy on the prevalence of ventilator-associated pneumonia in patients requiring long-term ventilatory care. Crit Care Med 30: 1983-1986, 2002.

5. Baker AM, Meredith JW and Haponik EF: Pneumonia in intubated trauma patients. Microbiology and outcomes. Am J Respir Crit Care Med 153: 343-349, 1996.

6. Guidelines for the management of adults with hospital-acquired, ventilator-associated, and healthcare-associated pneumonia. Am J Respir Crit Care Med 171: 388-416, 2005.

7. Magnotti LJ, Croce MA, Zarzaur BL, et al: Causative pathogen dictates optimal duration of antimicrobial therapy for ventilator-associated pneumonia in trauma patients. J Am Coll Surg 212: 476-484; discussion 484-486, 2011.

8. Memish ZA, Shibl AM, Kambal AM, Ohaly YA, Ishaq A and Livermore DM: Antimicrobial resistance among non-fermenting gram-negative bacteria in Saudi Arabia. J Antimicrob Chemother 67: 1701-1705, 2012.

9. Lee HY, Chen CL, Wang SB, et al: Imipenem heteroresistance induced by imipenem in multidrug-resistant Acinetobacter baumannii: mechanism and clinical implications. Int $\mathrm{J}$ Antimicrob Agents 37: 302-308, 2011.

10. Zavascki AP, Carvalhaes CG, Picão RC and Gales AC: Multidrug-resistant Pseudomonas aeruginosa and Acinetobacter baumannii: resistance mechanisms and implications for therapy. Expert Rev Anti Infect Ther 8: 71-93, 2010.

11. Gómez-Garcés JL, Aracil B, Gil Y and Burillo A: Susceptibility of 228 non-fermenting gram-negative rods to tigecycline and six other antimicrobial drugs. J Chemother 21: 267-271, 2009.

12. Gales AC, Jones RN, Forward KR, Liñares J, Sader HS and Verhoef J: Emerging importance of multidrug-resistant Acinetobacter species and Stenotrophomonas maltophilia as pathogens in seriously ill patients: geographic patterns, epidemiological features, and trends in the SENTRY Antimicrobial Surveillance Program (1997-1999). Clin Infect Dis 32 (Suppl 2): S104-S113, 2001 
13. Borcan E, Ghită CM, Chifiriuc MC, Mărutescu L, Isar C and Lăzar V: Antibiotic resistance of gram negative bacilli strains isolated from the intensive care unit in Fundeni Clinical Institute, Bucharest, Romania. Roum Arch Microbiol Immunol 68: 228-234, 2009.

14. Lu Q, Yang J, Liu Z, Gutierrez C, Aymard G and Rouby JJ: Nebulized ceftazidime and amikacin in ventilator-associated pneumonia caused by Pseudomonas aeruginosa. Am J Respir Crit Care Med 184: 106-115, 2011

15. Oliveira LG, Luengo J, Caramori JC, Montelli AC, Cunha MD and Barretti P: Peritonitis in recent years: clinical findings and predictors of treatment response of 170 episodes at a single Brazilian center. Int Urol Nephrol: Feb 3, 2012 (Epub ahead of print).
16. Morrow BM, Mowzer R, Pitcher R and Argent AC: Investigation into the effect of closed-system suctioning on the frequency of pediatric ventilator-associated pneumonia in a developing country. Pediatr Crit Care Med 13: e25-e32, 2012.

17. Ak O, Batirel A, Ozer S and Colakoğlu S: Nosocomial infections and risk factors in the intensive care unit of a teaching and research hospital: a prospective cohort study. Med Sci Monit 17: PH29-34, 2011. 